\title{
Practical Algorithms for a Family of Waterfilling Solutions
}

\author{
Daniel Pérez Palomar, Member, IEEE, and Javier Rodríguez Fonollosa, Senior Member, IEEE
}

\begin{abstract}
Many engineering problems that can be formulated as constrained optimization problems result in solutions given by a waterfilling structure; the classical example is the capacity-achieving solution for a frequency-selective channel. For simple waterfilling solutions with a single waterlevel and a single constraint (typically, a power constraint), some algorithms have been proposed in the literature to compute the solutions numerically. However, some other optimization problems result in significantly more complicated waterfilling solutions that include multiple waterlevels and multiple constraints. For such cases, it may still be possible to obtain practical algorithms to evaluate the solutions numerically but only after a painstaking inspection of the specific waterfilling structure. In addition, a unified view of the different types of waterfilling solutions and the corresponding practical algorithms is missing.

The purpose of this paper is twofold. On the one hand, it overviews the waterfilling results existing in the literature from a unified viewpoint. On the other hand, it bridges the gap between a wide family of waterfilling solutions and their efficient implementation in practice; to be more precise, it provides a practical algorithm to evaluate numerically a general waterfilling solution, which includes the currently existing waterfilling solutions and others that may possibly appear in future problems.
\end{abstract}

Index Terms-Constrained optimization problems, MIMO transceiver, parallel channels, practical algorithms, waterfilling, waterpouring.

\section{INTRODUCTION}

$\mathbf{T}$ HE well-known classical waterfilling solution solves the problem of maximizing the mutual information between the input and the output of a channel composed of several subchannels (such as a frequency-selective channel, a time-varying channel, or a set of parallel subchannels arising from the use of multiple antennas at both sides of the link) with a global power constraint at the transmitter [1]-[3]. This capacity-achieving solution has the visual interpretation of pouring water over a surface given by the inverse of the subchannel gains, hence the name waterfilling or waterpouring (cf. Section II).

The capacity-achieving waterfilling solution has been considered in many works to design efficient communication

Manuscript received August 13, 2003; revised January 17, 2004. This work was supported in part by the Catalan Government (DURSI) under Grant 2001SGR 00268 and the Spanish Government (CICYT) under Grant TIC2003-05482. The associate editor coordinating the review of this paper and approving it for publication was Dr. Athina Petropulu.

D. P. Palomar was with the Department of Signal Theory and Communications, Technical University of Catalonia (UPC), Campus Nord UPC, 08034 Barcelona, Spain. He is now with the Department of Electrical Engineering, Princeton University, Princeton, NJ 08544 (e-mail: danielp@ princeton.edu).

J. R. Fonollosa is with the Department of Signal Theory and Communications, Technical University of Catalonia (UPC), Campus Nord UPC, 08034 Barcelona, Spain (e-mail: fono@gps.tsc.upc.es, javier.fonollosa @upc.es).

Digital Object Identifier 10.1109/TSP.2004.840816 systems [4]-[6]. There are, however, other completely different problems that result in similar waterfilling solutions. In particular, when the transmitter and receiver are jointly designed for communications through multiple-input multiple-output (MIMO) channels, these types of solutions typically appear [7]. Perhaps the most popular of such problems is the minimization of the sum of the mean square errors (MSEs) (equivalently, the trace of the MSE matrix) of the different subchannels existing within a MIMO channel, resulting in a waterfilling solution [6]-[12] (for frequency-selective single-input single-output (SISO) channels, similar solutions were obtained already in the 1960s [13]). If, instead, the system is designed to minimize the determinant of the MSE matrix, the classical capacity-achieving waterfilling result is again obtained [12], [14] (this is due to the direct relation between the determinant of the MSE matrix and the mutual information [15]). The maximization of the minimum signal to interference-plus-noise ratio (SINR) among the subchannels also results in a waterfilling solution [7]. In [16], the minimization of the average bit error rate (BER) over a set of parallel subchannels was extensively treated obtaining a waterfilling result. In [17], minimum BER solutions were obtained for the case of imperfect channel knowledge at the transmitter, with a waterfilling power allocation. Recently, the problem of joint transmit-receive design to achieve minimum average BER in MIMO channels (with equal constellations) has been solved independently in [7] and [18], obtaining a solution that includes the same waterfilling structure as in the minimization of the trace of the MSE matrix.

The previous waterfilling solutions are very simple to evaluate because all of them have a single waterlevel and a power constraint. As a consequence, it is quite straightforward to compute them numerically in practice. Since the solution is parameterized with a single waterlevel, the problem reduces to obtaining the waterlevel such that the power constraint is satisfied with equality. In order to find the exact value of the waterlevel, different algorithms have been proposed that can be classified into iterative algorithms and exact algorithms. The iterative algorithms are trivially implemented in practice and get close to the exact value as the number of iterations goes to infinity [10], [13], [16], [19]. The exact algorithms give the exact value of the solution in a finite number of loops or iterations [5], [11], [20]-[22].

As opposed to the aforementioned simple waterfilling solutions, other design criteria, such as the minimization of the maximum of the BERs of the subchannels or the maximization of the harmonic mean of the SINRs of the subchannels, result in significantly more complicated waterfilling solutions with multiple waterlevels and multiple constraints (not just a 
simple power constraint) [7]. The minimum power design in an MIMO point-to-point communication system that satisfies a set of quality-of-service requirements among the used subchannels also results in a waterfilling solution with multiple waterlevels and multiple constraints [23]. In such cases, it is not clear how to compute the numerical solution in practice-not even by adopting an iterative method; however, after a painstaking analysis of the specific structure of each waterfilling solution, it is still possible to obtain practical algorithms that give the numerical solution [24]. It is thus desirable to develop a general approach to deal with these complicated waterfilling solutions, as opposed to obtaining results tailored to each particular case.

This paper considers a general waterfilling solution (including multiple waterlevels and multiple constraints) and provides a general algorithm to compute numerically the solution in practice. In other words, the paper bridges the gap between a wide family of waterfilling solutions and their practical implementation. Such a framework not only encompasses the currently existing waterfilling results and algorithms, but it may also serve as a basis to obtain practical algorithms in future problems with a similar structure. To be more precise, as long as a waterfilling solution fits within the general model considered in this paper, it suffices to use the general algorithm (known to give the exact numerical solution) and particularize it to the specific case at hand, as opposed to having to analyze the structure of the specific waterfilling solution to obtain an algorithm (and to prove that it gives the exact solution).

The paper is structured as follows. In Section II, the signal model for the classical capacity-achieving waterfilling solution and for a completely general waterfilling solution are introduced. Section III overviews the existing algorithms for simple waterfilling solutions, and Section IV provides the main result of the paper: an algorithm to evaluate numerically a general multiwaterlevel, multiconstrained waterfilling solution. Section V then considers the particularization of the general algorithm to specific examples of practical interest. Finally, in Section VI, the conclusions are drawn.

\section{WATERFILLING SOLUTIONS}

The classical capacity-achieving waterfilling result is the solution to the following constrained optimization problem:

$$
\begin{aligned}
\max _{\left\{x_{i}\right\}} & \sum_{i=1}^{L} \log \left(1+x_{i} \lambda_{i}\right) \\
\text { s.t. } & \sum_{i=1}^{L} x_{i} \leq P_{T} \\
& x_{i} \geq 0, \quad 1 \leq i \leq L
\end{aligned}
$$

given by

$$
x_{i}=\left(\mu-\lambda_{i}^{-1}\right)^{+} \quad 1 \leq i \leq L
$$

where $(x)^{+} \triangleq \max (0, x), L$ is the number of subchannels, $\lambda_{i}$ is the gain of the $i$ th subchannel, and $\mu$ is the waterlevel chosen to satisfy the power constraint with equality $\sum_{i} x_{i}=P_{T}$.

As can be seen in Fig. 1, this capacity-achieving solution has the visual interpretation of pouring water over a surface (or

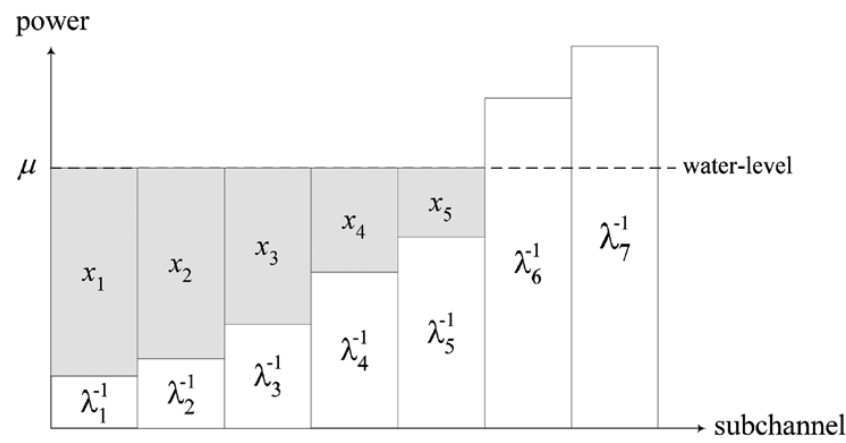

Fig. 1. Classical waterfilling power allocation $x_{i}=\left(\mu-\lambda_{i}^{-1}\right)^{+}$with $\sum_{i} x_{i}=P_{T}$

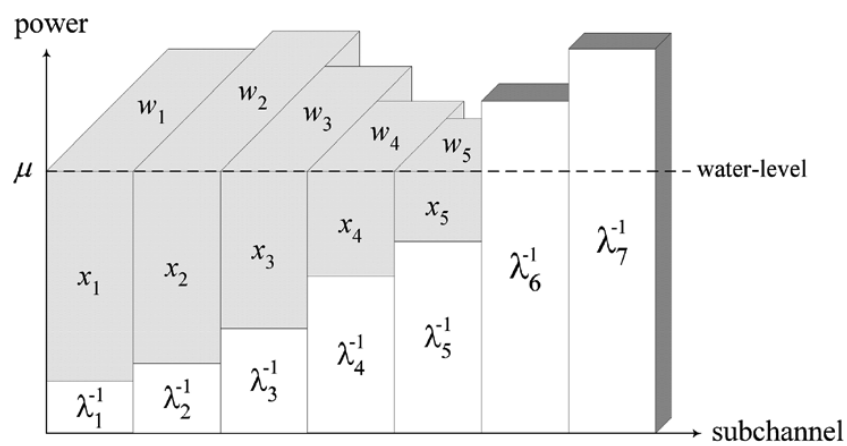

Fig. 2. General waterfilling power allocation $x_{i}=\left(\mu-\lambda_{i}^{-1}\right)^{+}$with $\sum_{i} x_{i} w_{i}=P_{T}$.

curve) given by the inverse of the subchannel gains, hence, the name waterfilling or waterpouring [1], [2]. More general waterfilling expressions such as $x_{i}=\left(\mu a_{i}-b_{i}\right)^{+}$, where the $a_{i}$ 's and $b_{i}$ 's are arbitrary positive numbers, also have the same visual interpretation after the change of variables $\tilde{x}_{i}=x_{i} / a_{i}, \tilde{\lambda}_{i}=$ $a_{i} / b_{i}$ and with a resulting weighted power constraint given by $\sum_{i} \tilde{x}_{i} w_{i}=P_{T}$, where $w_{i}=a_{i}$ are weights that can be visually interpreted as the width of each of the subchannels as can be observed from Fig. 2.

As has been previously mentioned, there are many other constrained optimization problems that result in similar waterfilling solutions, possibly with multiple waterlevels and multiple constraints. It would be of a great interest to deal simultaneously with all these waterfilling problems by developing a general framework that embraces a wide range of waterfilling solutions.

A very general multiwaterlevel multiconstrained waterfilling result can be defined as any solution of the form

$$
x_{k, i}=\left(\mu_{k} a_{k, i}-b_{k, i}\right)^{+}, \quad 1 \leq k \leq N, \quad 1 \leq i \leq L_{k}
$$

subject to a set of $M$ arbitrary equality (inequality) constraints

$$
f_{m}\left(\left\{\mu_{k}\right\}\right)=(\leq) 0, \quad 1 \leq m \leq M
$$

where the $\mu_{k}$ 's are the $N$ multiple waterlevels, the $x_{k, i}$ 's are the variables (there are $L_{k}$ variables associated with the same waterlevel $\mu_{k}$ ), the $a_{k, i}$ 's and the $b_{k, i}$ 's are given constant values, and the $f_{m}$ 's are arbitrary functions that constrain the waterlevels [note that constraints on the $x_{k, i}$ 's can be readily expressed as constraints on the $\mu_{k}$ 's simply by using the relation in (3)]. We denote by $L_{T}=\sum_{k=1}^{N} L_{k}$ the total number of subchannels. 
The numerical evaluation of the general waterfilling solution in (3) and (4) may be of a high complexity. In order to make the problem more tractable, we consider a less-general waterfilling solution by introducing an additional structure in the constraints. To be more precise, we consider $M=N+1$ constraints of the form

$$
\begin{aligned}
f_{k}\left(\mu_{k}\right) & =t, \quad 1 \leq k \leq N \\
g\left(\left\{\mu_{k}\right\}\right) & =0
\end{aligned}
$$

where the $f_{k}$ 's are monotonic functions that constrain each waterlevel independently, $g$ is a monotonic function that constrains all the waterlevels jointly, and $t$ is an additional auxiliary variable introduced for generality. It is important to remark that a waterfilling solution with constraints as in (5) is still very general and, in fact, includes all the waterfilling solutions previously considered in the literature (to the authors' knowledge). Note that it is not difficult to generalize the constraints considered in (5), at the expense of a more involved notation, to include more equality constraints at each $k$, inequality constraints at each $k$, a nonmonotonic global constraint $g$, more global equality constraints, and global inequality constraints. (The assumption of the $f_{k}$ 's being monotonic functions is necessary to reduce the complexity of the algorithm and make it practical.)

\section{Practical Algorithms for SimPle WATERFILLING SOLUTIONS}

For the simple case of single-waterlevel single-constrained waterfilling solutions, it is straightforward to obtain both iterative and exact algorithms. Iterative algorithms approach the exact solution as the number of iterations goes to infinity (in practice, of course, it suffices to stop iterating when the error is below a certain tolerance threshold). Exact algorithms give the exact result in a finite (and small) number of loops or iterations (with a worst-case complexity linear in the number of subchannels). The algorithms are based on the obvious fact that the solution is readily obtained once the waterlevel is known; therefore, the problem reduces to obtaining the waterlevel such that the constraint (typically, a power constraint) is satisfied.

Iterative algorithms can be straightforwardly obtained simply by fixing the waterlevel $\mu$ to some value and then adjusting it iteratively until the constraint is satisfied [19], [10], [16] (in [13], a parametric approach was proposed to obtain the curve of power versus performance, which also reduces to an iterative method when having to meet a specific power constraint). This is nothing more than the well-known problem of finding the root of a function (see Fig. 3), which happens to be nonlinear and nondifferentiable (a power constraint, for example, would correspond to the constraint function $g(\mu)=\sum_{i} x_{i}(\mu)-P_{T}$, where the dependence of the $x_{i}$ 's on the waterlevel $\mu$ has been made explicit). This can be done, for example, by modifying the waterlevel with small and (possibly) decreasing steps [10], [16] or by bisection until the error is below some tolerance threshold.

Exact algorithms can also be easily derived based on hypothesis testing. The underlying idea is to form a hypothesis of the active $\left(x_{i}>0\right)$ and inactive $\left(x_{i}=0\right)$ subchannels and check whether a consistent solution can be found conditioned on the

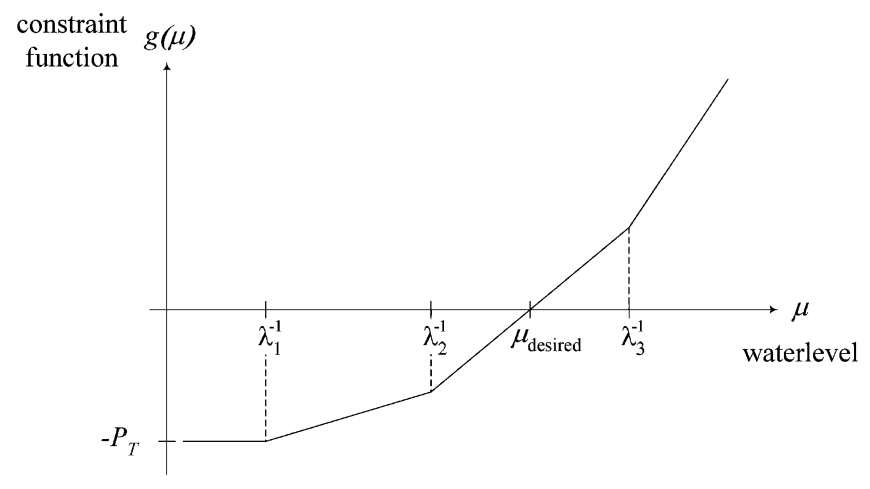

Fig. 3. Illustration of the waterlevel determination as a problem of finding the root of the constraint function $g(\mu)=\sum_{i} x_{i}(\mu)-P_{T}$, where $x_{i}=(\mu-$ $\left.\lambda_{i}^{-1}\right)^{+}$, and the $\lambda_{i}$ 's are in decreasing order.

hypothesis. In principle, such an approach has an exponential worst-case complexity of $2^{L}$, where $L$ is the number of subchannels; a careful inspection of the structure of the problem, however, reveals that it is possible to obtain a linear worst-case complexity of $L$. In [20, ch. 4] (see also [21, ch. 7]) and [5, Alg. 1], an exact algorithm was obtained for the classical capacity-achieving solution. In [11], a similar algorithm was also derived for the minimization of the weighted sum of the MSEs of the subchannels. In a first attempt to unify the waterfilling solutions and their corresponding algorithms, practical algorithms were proposed in [22] for general waterfilling solutions with a single waterlevel and a power constraint (single constraint).

The following section is devoted to obtaining an exact unified algorithm for more general multiwaterlevel multiconstrained waterfilling solutions.

\section{UNIFIED PRACTICAL Algorithm FOR A FAMILY OF WATERFILLING SOLUTIONS}

This section provides the main result of the paper: an exact algorithm for the numerical evaluation of a general waterfilling solution with multiple waterlevels and multiple constraints, as defined in (3) and (5). The interest of the following general algorithm is twofold. Not only it is able to evaluate numerically a wide family of waterfilling solutions, but it is also an exact algorithm as opposed to iterative methods that approach the desired solution as the number of iterations goes to infinity (cf. Section III).

Proposition 1: Suppose that a constrained optimization problem has a waterfilling solution of the form

$$
x_{k, i}=\left(\mu_{k} a_{k, i}-b_{k, i}\right)^{+}, \quad 1 \leq k \leq N, \quad 1 \leq i \leq L_{k}
$$

subject to the constraints

$$
\begin{aligned}
f_{k}\left(\mu_{k}\right) & =t, \quad 1 \leq k \leq N \\
g\left(\left\{\mu_{k}\right\}\right) & =0
\end{aligned}
$$

where the $\mu_{k}$ 's are the $N$ multiple waterlevels, the $x_{k, i}$ 's are the variables (there are $L_{k}$ variables associated with the same waterlevel $\left.\mu_{k}\right), t$ is an auxiliary variable, the $a_{k, i}$ 's and $b_{k, i}$ 's are given positive ${ }^{1}$ constant values, and the functions $f_{k}$ 's and $g$

\footnotetext{
${ }^{1}$ The values $a_{k, i}$ 's and $b_{k, i}$ 's are assumed positive for simplicity of exposi-
} tion. However, other cases can be similarly considered. 
are strictly increasing (the monotonicity can be nonstrict in the range of $\mu_{k}$ 's corresponding to all $x_{k, i}$ equal to zero for some $k$ ). Then, the waterfilling solution can be numerically evaluated in practice with Algorithm 1 with a worst-case complexity of $L_{T}=\sum_{k=1}^{N} L_{k}$ iterations, i.e., a linear complexity in the total number of subchannels.

Algorithm 1-Practical algorithm for the family of waterfilling solutions with multiple waterlevels and multiple constraints, as described in Proposition 1:

Input: Set of pairs $\left\{\left(a_{k, i}, b_{k, i}\right)\right\}$ and constraint functions $\left\{f_{k}\right\}$ and $g$.

Output: Numerical solution $\left\{x_{k, i}\right\}$ and set of waterlevels $\left\{\mu_{k}\right\}$.

0. Set $\tilde{L}_{k}=L_{k}$ for $1 \leq k \leq N$ and (if necessary) sort the set of pairs $\left\{\left(a_{k, i}, b_{k, i}\right)\right\}$ such that $\left\{a_{k, i} / b_{k, i}\right\}_{i=1}^{L_{k}}$ are in decreasing order $a_{k, i} / b_{k, i} \geq a_{k, i+1} / b_{k, i+1}$ for each $k$ (define $a_{k, L_{k}+1} / b_{k, L_{k}+1} \triangleq 0$ ).

1. Compute

$$
\begin{aligned}
t^{\mathrm{ub}} & =\min _{1 \leq k \leq N}\left\{f_{k}\left(b_{k, \tilde{L}_{k}+1} / a_{k, \tilde{L}_{k}+1}\right)\right\} \quad \text { and } \\
t^{\mathrm{lb}} & =\max _{1 \leq k \leq N}\left\{f_{k}\left(b_{k, \tilde{L}_{k}} / a_{k, \tilde{L}_{k}}\right)\right\}
\end{aligned}
$$

(denote the maximizing $k$ by $k_{\max }$ ).

2. If $t^{\mathrm{lb}}<t^{\mathrm{ub}}$ and $g\left(\left\{\mu_{k}\left(t^{\mathrm{lb}}\right)\right\}\right)<0$, where $\mu_{k}(t)=$ $f_{k}^{-1}(t),{ }^{2}$ then accept the hypothesis, and go to step 3 .

Otherwise, reject the hypothesis, form a new one by setting $\tilde{L}_{k_{\max }}=\tilde{L}_{k_{\max }}-1$, and go to step 1 .

3. Find $t_{0} \in\left(t^{\mathrm{lb}}, t^{\mathrm{ub}}\right] \mid g\left(\left\{\mu_{k}\left(t_{0}\right)\right\}\right)=0$, obtain the waterlevels and the numerical solution as

$$
\begin{aligned}
\mu_{k} & =f_{k}^{-1}\left(t_{0}\right), \quad 1 \leq k \leq N \\
x_{k, i} & =\left(\mu_{k} a_{k, i}-b_{k, i}\right)^{+}, \quad 1 \leq i \leq L_{k}
\end{aligned}
$$

undo the sorting done at step 0 (if any), and finish.

Note that the algorithm requires the sequences $a_{k, i}$ and $b_{k, i}$ properly sorted. The complexity of the best sorting algorithm is $O\left(N \log _{2} N\right)$ [25], where $N$ denotes the length of the sequences; however, in many applications, such sequences are already sorted. This is because they come from the eigenvalues of a matrix and many of the algorithms to compute the eigenvalues and eigenvectors already produce the eigenvalues sorted [25], [26]. In any case, the complexity of sorting the eigenvalues is insignificant compared with the complexity $O\left(N^{3}\right)$ to compute the eigenvectors so that we can always assume the eigenvalues sorted.

Note also that, strictly speaking, we can only claim that the algorithm obtains the exact numerical solution when the equation $g\left(\left\{\mu_{k}\left(t_{0}\right)\right\}\right)=0$ in the last step can be solved for $t_{0}$ in an exact way, e.g., when it can be solved in closed-form. Otherwise, some iterative method has to be used. In practice, however, this is not a problem since it is just a one-dimensional search that

\footnotetext{
${ }^{2}$ As stated in Proposition 1, the functions $f_{k}$ need not be strictly monotone for the range of $\mu_{k}$ in which all $x_{k, i}$ are zero for a given $k$. In such a case, we define the inverse as $f_{k}^{-1}(t) \triangleq \inf \left\{\mu_{k}: f_{k}\left(\mu_{k}\right)=t\right\}$ (this choice guarantees the minimum value of the function $g$ ).
}

is done just once (after the algorithm has obtained the optimum hypothesis). Therefore, such cases are also contemplated (see, for example, Algorithm 3 in Section V-B1).

Proof of Proposition 1: Algorithm 1 is based on hypothesis testing. First, it makes the hypothesis that all subchannels are active $\left(x_{k, i}>0, \forall k, i\right)$ and checks whether there is such a solution that satisfies all the constraints. If not, the hypothesis is rejected, a new hypothesis is made (properly chosen), and so forth. In principle, the total number of hypotheses is $2^{L_{T}}$ (since each subchannel can be either active $x_{k, i}>0$ or inactive $x_{k, i}=0$ ), which implies an exponential worst-case complexity in the total number of subchannels. However, after a careful inspection of the problem structure, it turns out that the worst-case complexity can be made linear.

For each hypothesis, there is a set of possible values for the auxiliary variable $t$ and, correspondingly, a set of possible values for the waterlevel $\mu_{k}$ 's. The problem is then to find the optimal hypothesis such that the waterlevels can be chosen to satisfy all the constraints. Interestingly, it is possible to obtain the exact way in which the hypotheses should be made such that the worst-case complexity is linear, starting from the hypothesis of all subchannels active and successively deactivating subchannels.

See the Appendix for a detailed proof.

Since many optimization problems result in waterfilling solutions with a single waterlevel and a single constraint, it is interesting to particularize the previous result to this case.

Corollary 1: Suppose that a constrained optimization problem has a waterfilling solution of the form

$$
x_{i}=\left(\mu a_{i}-b_{i}\right)^{+}, \quad 1 \leq i \leq L
$$

subject to the constraint

$$
g(\mu)=0
$$

where $\mu$ is the waterlevel, the $x_{i}$ 's are the $L$ variables, the $a_{i}$ 's and $b_{i}$ 's are given positive constant values, and the function $g$ is strictly increasing (the monotonicity can be nonstrict in the range of $\mu$ corresponding to all $x_{i}$ equal to zero). Then, the waterfilling solution can be numerically evaluated in practice with Algorithm 2 with a worst-case complexity of $L$ iterations, i.e., a linear complexity in the number of subchannels. ${ }^{3}$

Algorithm 2-Practical algorithm for the family of singlelevel waterfilling solutions, as described in Corollary 1:

Input: Set of pairs $\left\{\left(a_{i}, b_{i}\right)\right\}$ and constraint function $g$.

Output: Numerical solution $\left\{x_{i}\right\}$ and waterlevel $\mu$.

0 . Set $\tilde{L}=L$, and (if necessary) sort the set of pairs $\left\{\left(a_{i}, b_{i}\right)\right\}$ such that $a_{i} / b_{i}$ are in decreasing order $a_{i} / b_{i} \geq a_{i+1} / b_{i+1}$ (define $a_{L+1} / b_{L+1} \triangleq 0$ ).

1. If $b_{\tilde{L}} / a_{\tilde{L}}<b_{\tilde{L}+1} / a_{\tilde{L}+1}$ and $g\left(b_{\tilde{L}} / a_{\tilde{L}}\right)<0$, then accept the hypothesis, and go to step 2 .

Otherwise, reject the hypothesis, form a new one by setting $\tilde{L}=\tilde{L}-1$, and go to step 1 .

\footnotetext{
${ }^{3}$ It is also possible to find the correct hypothesis following a bisection approach with a complexity of $\log _{2}(L)$.
} 
2. Find the waterlevel $\mu \in\left(b_{\tilde{L}} / a_{\tilde{L}}, b_{\tilde{L}+1} / a_{\tilde{L}+1}\right] \mid g(\mu)=$ 0 , obtain the numerical solution as

$$
x_{i}=\left(\mu a_{i}-b_{i}\right)^{+}, \quad 1 \leq i \leq L
$$

undo the sorting done at step 0 (if any), and finish.

Proof of Corollary 1: The proof follows directly from Proposition 1 and Algorithm 1 by introducing the innocuous constraint given by the function $f(\mu)=\mu$, from which we ob$\operatorname{tain} t^{\mathrm{lb}}=b_{\tilde{L}} / a_{\tilde{L}}$ and $t^{\mathrm{ub}}=b_{\tilde{L}+1} / a_{\tilde{L}+1}$. Note that Algorithm 2 reduces to that obtained in [22] for the particular case of having a power constraint $g(\mu)=\sum_{i} x_{i}-P_{T}$.

Summarizing, we can say that for any constrained optimization problem with a solution that fits into the general waterfilling structure as defined in Proposition 1 (or Corollary 1), it is not necessary to struggle to obtain heuristic and iterative methods to compute the solution numerically in practice. It suffices to particularize Algorithm 1 (or Algorithm 2) with the guaranty that it will give the exact numerical solution with a linear worst-case complexity in the number of subchannels.

\section{PARTICULARIZATION TO DifFERENT EXAMPLES OF WATERFILLING SOLUTIONS}

In this section, we show how the general algorithms obtained in Section IV for a wide family of waterfilling solutions can be readily particularized to several cases of interest in the design of communication systems.

\section{A. Waterfilling Solutions With a Single Waterlevel}

To start with, we consider some examples of simple waterfilling solutions with a single waterlevel and a single constraint and show how Algorithm 2 can be easily particularized.

1) Maximization of the Mutual Information Subject to a Power Constraint: In communication systems, the maximization of the mutual information is a problem of a great interest since it gives the solution that achieves the channel capacity [1]-[5]. Such a capacity-achieving solution has a waterfilling form given by

$$
x_{i}=\left(\mu-\lambda_{i}^{-1}\right)^{+}, \quad 1 \leq i \leq L
$$

subject to the power constraint

$$
\sum_{i=1}^{L} x_{i}=P_{T}
$$

where $x_{i}$ is the power allocated to the $i$ th subchannel, with gain $\lambda_{i}$, and $P_{T}$ is the power available for transmission.

To obtain a practical algorithm, it suffices to use Algorithm 2 (single waterlevel and single constraint) introducing the following particularizations:

$$
\begin{aligned}
a_{i} & =1, \quad b_{i}=\lambda_{i}^{-1} \\
g(\mu) & =\mu \tilde{L}-\sum_{i=1}^{\tilde{L}} \lambda_{i}^{-1}-P_{T} .
\end{aligned}
$$

The comparison $g\left(b_{\tilde{L}} / a_{\tilde{L}}\right)<0$ reduces to $\lambda_{\tilde{L}}^{-1}<\left(P_{T}+\right.$ $\left.\sum_{i=1}^{\tilde{L}} \lambda_{i}^{-1}\right) / \tilde{L}$, and $\mu \mid g(\mu)=0$ is obtained as $\mu=\left(P_{T}+\right.$ $\left.\sum_{i=1}^{L} \lambda_{i}^{-1}\right) / \tilde{L}$.

2) Minimization of the Sum of the MSEs Subject to a Power Constraint: When the transmitter and receiver of a MIMO communication system are jointly designed to minimize the sum of the MSEs of the different subchannels (equivalently, the trace of the MSE matrix), the following waterfilling solution arises in the determination of the power distribution over the subchannels at the transmitter [6]-[11][19]:

$$
x_{i}=\left(\mu \lambda_{i}^{-1 / 2}-\lambda_{i}^{-1}\right)^{+}, \quad 1 \leq i \leq L
$$

subject to the power constraint

$$
\sum_{i=1}^{L} x_{i}=P_{T}
$$

where $x_{i}$ is the power allocated to the $i$ th subchannel, with gain $\lambda_{i}$, and $P_{T}$ is the power available for transmission.

To obtain a practical algorithm, it suffices to use Algorithm 2 (single waterlevel and single constraint) introducing the following particularizations:

$$
\begin{aligned}
a_{i} & =\lambda_{i}^{-1 / 2}, \quad b_{i}=\lambda_{i}^{-1} \\
g(\mu) & =\mu \sum_{i=1}^{\tilde{L}} \lambda_{i}^{-1 / 2}-\sum_{i=1}^{\tilde{L}} \lambda_{i}^{-1}-P_{T} .
\end{aligned}
$$

The comparison $g\left(b_{\tilde{L}} / a_{\tilde{L}}\right)<0$ reduces to $\lambda_{\tilde{L}}^{-1 / 2}<\left(P_{T}+\right.$ $\left.\sum_{i=1}^{\tilde{L}} \lambda_{i}^{-1}\right) / \sum_{i=1}^{\tilde{L}} \lambda_{i}^{-1 / 2}$, and $\mu \mid g(\mu)=0$ is obtained as $\mu=$ $\left(P_{T}+\sum_{i=1}^{L} \lambda_{i}^{-1}\right) / \sum_{i=1}^{\tilde{L}} \lambda_{i}^{-1 / 2}$.

3) Minimization of the Transmit Power Subject to Equal MSE Constraints: Similarly to Section V-A2, the joint design of the transmitter and receiver of a MIMO communication system to minimize the transmit power subject to equal MSE constraints for the different subchannels yields a waterfilling power allocation over the subchannels at the transmitter [23] given by

$$
x_{i}=\left(\mu \lambda_{i}^{-1 / 2}-\lambda_{i}^{-1}\right)^{+}, \quad 1 \leq i \leq L
$$

subject to the constraint

$$
\sum_{i=1}^{L} \frac{1}{1+x_{i} \lambda_{i}}=\rho
$$

where $x_{i}$ is the power allocated to the $i$ th subchannel, with gain $\lambda_{i}$, and $\rho / L$ is the MSE required at each subchannel.

To obtain a practical algorithm, it suffices to use Algorithm 2 (single waterlevel and single constraint) introducing the following particularizations:

$$
\begin{aligned}
a_{i} & =\lambda_{i}^{-1 / 2}, \quad b_{i}=\lambda_{i}^{-1} \\
g(\mu) & =-\left((L-\tilde{L})+\mu^{-1} \sum_{i=1}^{\tilde{L}} \lambda_{i}^{-1 / 2}-\rho\right) .
\end{aligned}
$$


Note the additional sign in the definition of function $g$ to make it increasing with respect to $\mu$, as required in Corollary 1 . The comparison $g\left(b_{\tilde{L}} / a_{\tilde{L}}\right)<0$ reduces to $\lambda_{\tilde{L}}^{-1 / 2}<\left(\sum_{i=1}^{\tilde{L}} \lambda_{i}^{-1 / 2}\right) /(\rho-(L-\tilde{L}))$, and $\mu \mid g(\mu)=0$ is obtained as $\mu=\left(\sum_{i=1}^{\tilde{L}} \lambda_{i}^{-1 / 2}\right) /(\rho-(L-\tilde{L}))$.

4) Minimization of the Average BER Subject to a Power Constraint: Since the BER is the ultimate performance measure of digital communications (rather than the MSE or SINR), the minimization of the BER is perhaps the most reasonable criterion to design a communication system. In [27], an OFDM system was optimized to minimize the BER averaged over the carriers; to be more precise, the waterfilling solution $x_{i}=\left(\mu \lambda_{i}^{1 /(m+1)}\right.$ $1)^{+} m / \lambda_{i}$ was obtained for the case with only statistic channel knowledge (following a Nakagami- $m$ pdf) and $x_{i}=(\mu+$ $\left.\log \lambda_{i}\right)^{+} / \lambda_{i}$ for perfect channel knowledge. Similarly, in [16], a parallel multiantenna MIMO system with minimum average BER was also considered with perfect channel knowledge and with additional constraints to guarantee a minimum BER per subchannel, obtaining a waterfilling solution with multiple waterlevels and multiple constraints, which is briefly considered in Section V-B3. However, after approximating the BER function with the Chernoff upper bound, the solution reduces to the following simple waterfilling solution, which we now further develop for illustration purposes (after an appropriate change of notation with respect to $[16,(28)])$ :

subject to

$$
x_{i}=\left(\mu-\left(c_{i}-\log \lambda_{i}\right)\right)^{+}, \quad 1 \leq i \leq L
$$

$$
\sum_{i=1}^{L}\left(x_{i}+c_{i}\right) / \lambda_{i}=P_{T}
$$

where the term $\left(x_{i}+c_{i}\right) / \lambda_{i}$ denotes the power allocated to the $i$ th subchannel, with gain $\lambda_{i}, c_{i}$ is the the minimum SINR required on the $i$ th subchannel (to guarantee a minimum BER), and $P_{T}$ is the power available for transmission.

To obtain a practical algorithm, it suffices to use Algorithm 2 (single waterlevel and single constraint), introducing the following particularizations: ${ }^{4}$

$$
\begin{aligned}
a_{i} & =1, \quad b_{i}=c_{i}-\log \lambda_{i} \\
g(\mu) & =\sum_{i=1}^{\tilde{L}}\left(\mu+\log \lambda_{i}\right) / \lambda_{i}+\sum_{i=1+\tilde{L}}^{L} c_{i} / \lambda_{i}-P_{T} .
\end{aligned}
$$

\section{B. Waterfilling Solutions With Multiple Waterlevels}

We now turn into more complicated waterfilling solutions with multiple waterlevels and multiple constraints and show how Algorithm 1 can be easily particularized.

1) Minimization of the Maximum of the MSEs Subject to a Power Constraint: The joint design of the transmitter and receiver to minimize the maximum of the MSEs of a communication through a multicarrier multiantenna MIMO channel has

\footnotetext{
${ }^{4}$ To be exact, we have to add a constant value to each term $c_{i}-\log \lambda_{i}$ to make sure that the $b_{i}$ 's are positive values. Such an approach, however, simplifies to the given expressions.
}

been shown to be an excellent criterion in terms of global performance since it guarantees the performance of the worst of the subchannels [7]. The corresponding waterfilling solution is

$$
x_{k, i}=\left(\mu_{k} \lambda_{k, i}^{-1 / 2}-\lambda_{k, i}^{-1}\right)^{+}, \quad 1 \leq k \leq N, \quad 1 \leq i \leq L_{k}
$$

subject to the constraints

$$
\begin{aligned}
& \frac{1}{\check{L}_{k}}\left(\left(\check{L}_{k}-L_{k}\right)+\sum_{i=1}^{L_{k}} \frac{1}{1+x_{k, i} \lambda_{k, i}}\right)=t, 1 \leq k \leq N \\
& \sum_{k=1}^{N} \sum_{i=1}^{L_{k}} x_{k, i}=P_{T}
\end{aligned}
$$

where $k$ and $i$ denote the carrier and the spatial eigenmode, respectively, $N$ is the number of carriers, $L_{k}$ is the number of used spatial eigenmodes at the $k$ th carrier, $x_{k, i}$ is the power allocated to the $(k, i)$ th subchannel, with gain $\lambda_{k, i}$, the $\breve{L}_{k}$ 's are given values, $t$ is an auxiliary variable to be determined, and $P_{T}$ is the power available for transmission (see [7] for details).

Since the waterfilling solution has multiple waterlevels and multiple constraints, Algorithm 1 can be used to obtain a practical algorithm with the following particularizations:

$$
\begin{aligned}
a_{k, i} & =\lambda_{k, i}^{-1 / 2}, \quad b_{k, i}=\lambda_{k, i}^{-1} \\
f_{k}\left(\mu_{k}\right) & =-\frac{1}{\breve{L}_{k}}\left(\left(\check{L}_{k}-\tilde{L}_{k}\right)+\mu_{k}^{-1} \sum_{i=1}^{\tilde{L}_{k}} \lambda_{k, i}^{-1 / 2}\right) \\
g\left(\left\{\mu_{k}\right\}\right) & =\sum_{k=1}^{N} \mu_{k} \sum_{i=1}^{\tilde{L}_{k}} \lambda_{k, i}^{-1 / 2}-\sum_{k=1}^{N} \sum_{i=1}^{\tilde{L}_{k}} \lambda_{k, i}^{-1}-P_{T} .
\end{aligned}
$$

Note the additional sign in the definition of the $f_{k}$ 's to make them increasing with respect to the $\mu_{k}$ 's as required in Proposition 1. From $f_{k}\left(\mu_{k}\right)=t$, it follows that

$$
\mu_{k}=\frac{\sum_{i=1}^{\tilde{L}_{k}} \lambda_{k, i}^{-1 / 2}}{-t \check{L}_{k}-\left(\check{L}_{k}-\tilde{L}_{k}\right)}
$$

and then

$$
g(t)=\sum_{k=1}^{N} \frac{\left(\sum_{i=1}^{\tilde{L}_{k}} \lambda_{k, i}^{-1 / 2}\right)^{2}}{-t \check{L}_{k}-\left(\check{L}_{k}-\tilde{L}_{k}\right)}-\sum_{k=1}^{N} \sum_{i=1}^{\tilde{L}_{k}} \lambda_{k, i}^{-1}-P_{T} .
$$

The general algorithm then reduces to the following one (in [24], a similar algorithm was derived for this specific problem).

Algorithm 3-Practical algorithm for the minimization of the maximum of the MSEs subject to a power constraint:

Input: Set of subchannel gains $\left\{\lambda_{k, i}\right\}$ and maximum power $P_{T}$.

Output: Numerical solution $\left\{x_{k, i}\right\}$ and set of waterlevels $\left\{\mu_{k}\right\}$.

0 . Set $\tilde{L}_{k}=L_{k}$ for $1 \leq k \leq N$ and (if necessary) sort the set of gains $\left\{\lambda_{k, i}\right\}$ such that $\left\{\lambda_{k, i}\right\}_{i=1}^{L_{k}}$ are in decreasing order $\lambda_{k, i} \geq \lambda_{k, i+1}$ for each $k$ (define $\lambda_{k, L_{k}+1} \triangleq 0$ ). 
1. Compute

$t^{\mathrm{ub}}=-\max _{1 \leq k \leq N}\left\{\frac{1}{\check{L}_{k}}\left(\left(\check{L}_{k}-\tilde{L}_{k}\right)+\lambda_{k, \tilde{L}_{k}+1}^{1 / 2} \sum_{i=1}^{\tilde{L}_{k}} \lambda_{k, i}^{-1 / 2}\right)\right\}$ and

$t^{\mathrm{lb}}=-\min _{1 \leq k \leq N}\left\{\frac{1}{\check{L}_{k}}\left(\left(\check{L}_{k}-\tilde{L}_{k}\right)+\lambda_{k, \tilde{L}_{k}}^{1 / 2} \sum_{i=1}^{\tilde{L}_{k}} \lambda_{k, i}^{-1 / 2}\right)\right\}$

(denote the minimizing $k$ by $k_{\text {min }}$ ).

2. If $t^{\mathrm{lb}}<t^{\mathrm{ub}}$ and $\sum_{k=1}^{N}\left(\left(\sum_{i=1}^{\tilde{L}_{k}} \lambda_{k, i}^{-1 / 2}\right)^{2} /\left(-t^{\mathrm{lb}} \check{L}_{k}-\right.\right.$ $\left.\left.\left(\check{L}_{k}-\tilde{L}_{k}\right)\right)\right)<P_{T}+\sum_{k=1}^{N} \sum_{i=1}^{\tilde{L}_{k}} \lambda_{k, i}^{-1}$, then accept the hypothesis, and go to step 3 .

Otherwise, reject the hypothesis, form a new one by setting $\tilde{L}_{k_{\min }}=\tilde{L}_{k_{\min }}-1$, and go to step 1 .

3. Obtain $t_{0}$, the waterlevels, and the numerical solution as

$t_{0} \in\left(t^{\mathrm{lb}}, t^{\mathrm{ub}}\right]: \sum_{k=1}^{N} \frac{\left(\sum_{i=1}^{\tilde{L}_{k}} \lambda_{k, i}^{-1 / 2}\right)^{2}}{-t_{0} \check{L}_{k}-\left(\check{L}_{k}-\tilde{L}_{k}\right)}=P_{T}+\sum_{k=1}^{N} \sum_{i=1}^{\tilde{L}_{k}} \lambda_{k, i}^{-1}$

$\mu_{k}=\frac{\sum_{i=1}^{\tilde{L}_{k}} \lambda_{k, i}^{-1 / 2}}{-t_{0} \check{L}_{k}-\left(\check{L}_{k}-\tilde{L}_{k}\right)}, \quad 1 \leq k \leq N, \quad$ and

$x_{k, i}=\left(\mu_{k} \lambda_{k, i}^{-1 / 2}-\lambda_{k, i}^{-1}\right)^{+}, \quad 1 \leq k \leq N, \quad 1 \leq i \leq L_{k}$

undo the sorting done at step 0 (if any), and finish.

2) Maximization of the Harmonic Mean of the SINRs Subject to a Power Constraint: Similarly to Section V-B1, if the transmitter and receiver are jointly designed to maximize the harmonic mean of the SINRs of a communication through a MIMO channel, the following waterfilling solution is obtained [7]:

$$
x_{k, i}=\left(\mu_{k} \lambda_{k, i}^{-1 / 2}-\lambda_{k, i}^{-1}\right)^{+}, \quad 1 \leq k \leq N, \quad 1 \leq i \leq L_{k}
$$

subject to the constraints

$$
\begin{gathered}
\frac{\mu_{k}}{\check{L}_{k}^{1 / 2}\left(L_{k}-\sum_{i=1}^{L_{k}} \frac{1}{1+x_{k, i} \lambda_{k, i}}\right)}=t, \quad 1 \leq k \leq N \\
\sum_{k=1}^{N} \sum_{i=1}^{L_{k}} x_{k, i}=P_{T}
\end{gathered}
$$

where the notation is as in (19)-(21).

The waterfilling solution has multiple waterlevels and multiple constraints, and therefore, Algorithm 1 can be used to obtain a practical algorithm with the following particularizations:

$$
\begin{aligned}
a_{k, i} & =\lambda_{k, i}^{-1 / 2}, \quad b_{k, i}=\lambda_{k, i}^{-1} \\
f_{k}\left(\mu_{k}\right) & =\frac{1}{\check{L}_{k}^{1 / 2}}\left(\mu_{k} \tilde{L}_{k}-\sum_{i=1}^{\tilde{L}_{k}} \lambda_{k, i}^{-1 / 2}\right) \\
g\left(\left\{\mu_{k}\right\}\right) & =\sum_{k=1}^{N} \mu_{k} \sum_{i=1}^{\tilde{L}_{k}} \lambda_{k, i}^{-1 / 2}-\sum_{k=1}^{N} \sum_{i=1}^{\tilde{L}_{k}} \lambda_{k, i}^{-1}-P_{T} .
\end{aligned}
$$

From $f_{k}\left(\mu_{k}\right)=t$, it follows that

$$
\mu_{k}=\frac{1}{\tilde{L}_{k}}\left(t \check{L}_{k}^{1 / 2}+\sum_{i=1}^{\tilde{L}_{k}} \lambda_{k, i}^{-1 / 2}\right)
$$

and then

$$
\begin{aligned}
g(t)=t \sum_{k=1}^{N} \frac{\check{L}_{k}^{1 / 2}}{\tilde{L}_{k}} \sum_{i=1}^{\tilde{L}_{k}} \lambda_{k, i}^{-1 / 2} & \\
& +\sum_{k=1}^{N} \frac{1}{\tilde{L}_{k}}\left(\sum_{i=1}^{\tilde{L}_{k}} \lambda_{k, i}^{-1 / 2}\right)^{2}-\sum_{k=1}^{N} \sum_{i=1}^{\tilde{L}_{k}} \lambda_{k, i}^{-1}-P_{T} .
\end{aligned}
$$

The general algorithm reduces then to the following one (in [24], a similar algorithm was derived for this specific problem).

Algorithm 4-Practical algorithm for the maximization of the harmonic mean of the SINRs subject to a power constraint:

Input: Set of subchannel gains $\left\{\lambda_{k, i}\right\}$ and maximum power $P_{T}$.

Output: Numerical solution $\left\{x_{k, i}\right\}$ and set of waterlevels $\left\{\mu_{k}\right\}$.

0. Set $\tilde{L}_{k}=L_{k}$ for $1 \leq k \leq N$, and (if necessary) sort the set of gains $\left\{\lambda_{k, i}\right\}$ such that $\left\{\lambda_{k, i}\right\}_{i=1}^{L_{k}}$ are in decreasing order $\lambda_{k, i} \geq \lambda_{k, i+1}$ for each $k$ (define $\lambda_{k, L_{k}+1} \triangleq 0$ ).

1. Compute

$$
\begin{aligned}
t^{\mathrm{ub}} & =\min _{1 \leq k \leq N}\left\{\frac{1}{\check{L}_{k}^{1 / 2}}\left(\lambda_{k, \tilde{L}_{k}+1}^{-1 / 2} \tilde{L}_{k}-\sum_{i=1}^{\tilde{L}_{k}} \lambda_{k, i}^{-1 / 2}\right)\right\} \\
t^{\mathrm{lb}} & =\max _{1 \leq k \leq N}\left\{\frac{1}{\check{L}_{k}^{1 / 2}}\left(\lambda_{k, \tilde{L}_{k}}^{-1 / 2} \tilde{L}_{k}-\sum_{i=1}^{\tilde{L}_{k}} \lambda_{k, i}^{-1 / 2}\right)\right\}
\end{aligned}
$$
and

(denote the maximizing $k$ by $k_{\max }$ ).

2. If $t^{\mathrm{lb}}<t^{\mathrm{ub}}$ and $t^{\mathrm{lb}}<\left(P_{T}+\sum_{k=1}^{N}\left(\sum_{i=1}^{\tilde{L}_{k}} \lambda_{k, i}^{-1}-\right.\right.$ $\left.\left.\left(\sum_{i=1}^{\tilde{L}_{k}} \lambda_{k, i}^{-1 / 2}\right)^{2} / \tilde{L}_{k}\right)\right) /\left(\sum_{k=1}^{N} \check{L}_{k}^{1 / 2} / \tilde{L}_{k} \sum_{i=1}^{\tilde{L}_{k}} \lambda_{k, i}^{-1 / 2}\right)$, then accept the hypothesis, and go to step 3 .

Otherwise reject the hypothesis, form a new one by setting $\tilde{L}_{k_{\max }}=\tilde{L}_{k_{\max }}-1$, and go to step 1 .

3. Obtain $t_{0}$, the waterlevels, and the numerical solution as

$$
\begin{gathered}
t_{0}=\frac{P_{T}+\sum_{k=1}^{N}\left(\sum_{i=1}^{\tilde{L}_{k}} \lambda_{k, i}^{-1}-\frac{1}{\tilde{L}_{k}}\left(\sum_{i=1}^{\tilde{L}_{k}} \lambda_{k, i}^{-1 / 2}\right)^{2}\right)}{\sum_{k=1}^{N} \frac{\check{L}_{k}^{1 / 2}}{\tilde{L}_{k}} \sum_{i=1}^{\tilde{L}_{k}} \lambda_{k, i}^{-1 / 2}} \\
\mu_{k}=\frac{1}{\tilde{L}_{k}}\left(t_{0} \check{L}_{k}^{1 / 2}+\sum_{i=1}^{\tilde{L}_{k}} \lambda_{k, i}^{-1 / 2}\right), \quad 1 \leq k \leq N, \quad \text { and } \\
x_{k, i}=\left(\mu_{k} \lambda_{k, i}^{-1 / 2}-\lambda_{k, i}^{-1}\right)^{+}, 1 \leq k \leq N, \quad 1 \leq i \leq L_{k}
\end{gathered}
$$

undo the sorting done at step 0 (if any), and finish.

3) Minimization of the Average BER Subject to a Power Constraint: As previously mentioned in Section V-A4, the minimization of the BER averaged over the parallel subchannels existing within a MIMO channel results in a waterfilling solution with multiple waterlevels and multiple constraints [16] given by 


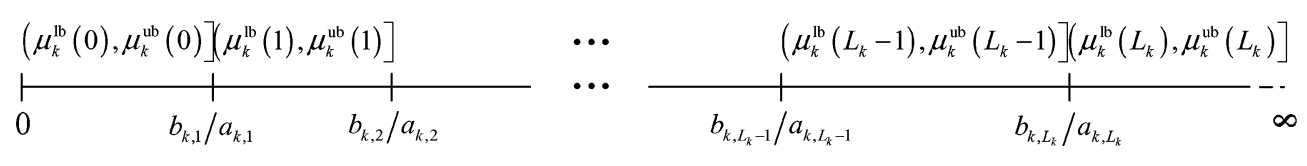

Fig. 4. Illustration of the partition of the allowable waterlevels as a function of the hypothesis for a given index $k$.

(after an appropriate change of notation with respect to [16, (25) and (26)])

$$
x_{k}=\left(\mu_{k}-\frac{c_{k}}{\lambda_{k}}\right)^{+}, \quad 1 \leq k \leq N
$$

subject to the constraints

$$
\begin{aligned}
\sqrt{\frac{\lambda_{k}}{\mu_{k}}} \exp \left(-\mu_{k} \lambda_{k}\right) & =t, \quad 1 \leq k \leq N \\
\sum_{k=1}^{N}\left(x_{k}+\frac{c_{k}}{\lambda_{k}}\right) & =P_{T}
\end{aligned}
$$

where the notation is as in (17) and (18), and $t$ is an auxiliary variable to be determined.

This waterfilling solution, although including multiple waterlevels, is still simple to solve in practice since each waterlevel $\mu_{k}$ has a single associated variable $x_{k}$, i.e., $L_{k}=1$. As proposed in [16], the numerical computation is straightforward simply by iteratively updating the auxiliary variable $t$ (from which the waterlevels $\mu_{k}$ 's and then the solution $x_{k}$ can be readily obtained) until the power constraint is satisfied (cf. Section III). Alternatively, we can use the general result of Section IV, i.e., Proposition 1 and Algorithm 1, to first obtain the optimum hypothesis and then the auxiliary variable $t_{0} \in\left(t^{\mathrm{lb}}, t^{\mathrm{ub}}\right] \mid g\left(\left\{\mu_{k}\left(t_{0}\right)\right\}\right)=$ 0 using an iterative approach, as described above. Therefore, for this particular waterfilling solution, the use of Algorithm 1 (properly particularized) is useful only to obtain the optimum hypothesis and a small initial interval $\left(t^{\mathrm{lb}}, t^{\mathrm{ub}}\right]$ known to contain the optimum $t$ to start the iterative search.

\section{CONCLUSION}

Many different constrained optimization problems result in waterfilling solutions for which some algorithms have been proposed in the literature to compute the numerical solution in practice.

This paper, instead of dealing with each specific waterfilling result, has considered a general waterfilling formulation with multiple waterlevels and multiple constraints, which embraces a wide family of cases and has obtained an algorithm that provides the numerical solution with a linear worst-case complexity in the number of subchannels. In order to obtain an algorithm to evaluate a specific waterfilling solution, it suffices to particularize the general algorithm obtained in this paper to the problem at hand without having to prove that such an algorithm indeed gives the desired solution with a linear worst-case complexity. In this sense, this paper bridges the gap between a wide family of waterfilling solutions and their efficient practical implementation.

\section{APPENDIX \\ PROOF OF PROPOSITION 1}

As previously mentioned in the overview of the proof, the total number of hypotheses is, in principle, $2^{L_{T}}$. However, as shown next, the worst-case complexity can be made linear. The first step in this simplification is obtained by sorting the set of pairs $\left\{\left(a_{k, i}, b_{k, i}\right)\right\}_{i=1}^{L_{k}}$ such that $\left\{a_{k, i} / b_{k, i}\right\}_{i=1}^{L_{k}}$ are in decreasing order for each $k$ (i.e., $a_{k, i} / b_{k, i} \geq a_{k, i+1} / b_{k, i+1}$ ). With this ordering and since $x_{k, i} / b_{k, i}=\left(\mu_{k} a_{k, i} / b_{k, i}-1\right)^{+}$, a hypothesis is completely described by the set of active subchannels $\left\{\tilde{L}_{k}\right\}$ (such that $x_{k, i}>0$ for $1 \leq k \leq N, 1 \leq i \leq \tilde{L}_{k}$ and zero otherwise). The total number of hypotheses has been reduced to $\prod_{k=1}^{N}\left(L_{k}+1\right)$ (since there are only $L_{k}+1$ possibilities for each $k$ ), which can be seen to be smaller than the initial exponential complexity $2^{\sum_{k} L_{k}}$ by rewriting it as $2^{\sum_{k} \log _{2}\left(L_{k}+1\right)}$ and noting that $\log _{2}\left(L_{k}+1\right) \leq L_{k}$. In the following, this complexity will be further reduced to $L_{T}$ by using the fact that the hypotheses of the different $k$ 's are coupled.

First of all, let us analyze in detail the waterlevels $\mu_{k}$ 's. Consider a hypothesis given by $\left\{\tilde{L}_{k}\right\}$. The waterlevels for such a hypothesis must be such that the considered $\tilde{L}_{k}$ subchannels are indeed active while the rest remain inactive:

$$
\begin{aligned}
\mu_{k} a_{k, i}-b_{k, i} \begin{cases}>0 & 1 \leq k \leq N, \quad 1 \leq i \leq \tilde{L}_{k} \\
\leq 0 & 1 \leq k \leq N, \quad \tilde{L}_{k}<i \leq L_{k}\end{cases} \\
\Longleftrightarrow \quad \mu_{k}^{\mathrm{lb}}\left(\tilde{L}_{k}\right)<\mu_{k} \leq \mu_{k}^{\mathrm{ub}}\left(\tilde{L}_{k}\right), \quad 1 \leq k \leq N
\end{aligned}
$$

where $\mu_{k}^{\mathrm{lb}}\left(\tilde{L}_{k}\right)=b_{k, \tilde{L}_{k}} / a_{k, \tilde{L}_{k}}$ and $\mu_{k}^{\mathrm{ub}}\left(\tilde{L}_{k}\right)=$ $b_{k, \tilde{L}_{k}+1} / a_{k, \tilde{L}_{k}+1}$ (for convenience of notation, we define $\mu_{k}^{\mathrm{lb}}(0) \triangleq 0$ and $\left.\mu_{k}^{\mathrm{ub}}\left(L_{k}\right) \triangleq \infty\right)$. Therefore, simply because of the nature of the waterfilling solution, the waterlevels are lower and upper bounded for a given hypothesis. Noting that $\mu_{k}^{\mathrm{ub}}\left(\tilde{L}_{k}\right)=\mu_{k}^{\mathrm{lb}}\left(\tilde{L}_{k}+1\right)$, it follows that the different hypotheses partition the set of waterlevels for a given $k$ (see Fig. 4):

$$
\begin{aligned}
& \bigcup_{\tilde{L}_{k}=0}^{L_{k}}\left(\mu_{k}^{\mathrm{lb}}\left(\tilde{L}_{k}\right), \mu_{k}^{\mathrm{ub}}\left(\tilde{L}_{k}\right)\right]=(0, \infty] \\
& \quad\left(\mu_{k}^{\mathrm{lb}}\left(\tilde{L}_{k}^{(i)}\right), \mu_{k}^{\mathrm{ub}}\left(\tilde{L}_{k}^{(i)}\right)\right] \\
& \bigcap\left(\mu_{k}^{\mathrm{lb}}\left(\tilde{L}_{k}^{(j)}\right), \mu_{k}^{\mathrm{ub}}\left(\tilde{L}_{k}^{(j)}\right)\right]=\varnothing, \tilde{L}_{k}^{(i)} \neq \tilde{L}_{k}^{(j)} .
\end{aligned}
$$

Since functions $f_{k}$ 's are strictly increasing, it follows from (28) that ${ }^{5}$

$$
f_{k}\left(\mu_{k}^{\mathrm{lb}}\left(\tilde{L}_{k}\right)\right)<f_{k}\left(\mu_{k}\right) \leq f_{k}\left(\mu_{k}^{\mathrm{ub}}\left(\tilde{L}_{k}\right)\right), \quad 1 \leq k \leq N .
$$

${ }^{5}$ We make an abuse of notation since the left inequality is nonstrict for $\tilde{L}_{k}=$ 0 , i.e., $f_{k}\left(\mu_{k}^{\mathrm{lb}}(0)\right) \leq f_{k}\left(\mu_{k}\right) \leq f_{k}\left(\mu_{k}^{\mathrm{ub}}(0)\right)$ for $1 \leq k \leq N$. 
In addition, due to the constraints $f_{k}\left(\mu_{k}\right)=t$ for $1 \leq k \leq N$, the following bounds on $t$ are obtained:

$$
t_{k}^{\mathrm{lb}}\left(\tilde{L}_{k}\right)<t \leq t_{k}^{\mathrm{ub}}\left(\tilde{L}_{k}\right), \quad 1 \leq k \leq N
$$

where $t_{k}^{\mathrm{lb}}\left(\tilde{L}_{k}\right)=f_{k}\left(\mu_{k}^{\mathrm{lb}}\left(\tilde{L}_{k}\right)\right)$, and $t_{k}^{\mathrm{ub}}\left(\tilde{L}_{k}\right)=f_{k}\left(\mu_{k}^{\mathrm{ub}}\left(\tilde{L}_{k}\right)\right)$. Note that the partition on the waterlevels of (29) (see Fig. 4) translates into a partition on $t$ for each $k$. More compactly, the previous set of bounds on $t$ can be rewritten as

$$
t^{\mathrm{lb}}\left(\left\{\tilde{L}_{k}\right\}\right)<t \leq t^{\mathrm{ub}}\left(\left\{\tilde{L}_{k}\right\}\right)
$$

where $t^{\mathrm{lb}}\left(\left\{\tilde{L}_{k}\right\}\right)=\max _{1 \leq k \leq N}\left\{t_{k}^{\mathrm{lb}}\left(\tilde{L}_{k}\right)\right\}$ (denote the maximizing $k$ by $\left.k_{\max }\right)$, and $t^{\mathrm{ub}}\left(\left\{\tilde{L}_{k}\right\}\right)=\min _{1 \leq k \leq N}\left\{t_{k}^{\mathrm{ub}}\left(\tilde{L}_{k}\right)\right\}$.

For a given hypothesis, we have bounded the set of possible values for $t$ and, correspondingly, for the waterlevels based on the form of the waterfilling solution $x_{k, i}=\left(\mu_{k} a_{k, i}-b_{k, i}\right)^{+}$ and on the constraints $f_{k}\left(\mu_{k}\right)=t$. To finally decide whether the hypothesis is accepted or rejected, we still have to check whether there is a set of waterlevels $\left\{\mu_{k}\right\}$ within the bounded set such that $g\left(\left\{\mu_{k}\right\}\right)=0$. It is convenient to rewrite $g$ as a function of $t$ (note that $\mu_{k}=f_{k}^{-1}(t)$ ) as

$$
g(t) \triangleq g\left(\left\{\mu_{k}(t)\right\}\right)=g\left(\left\{f_{k}^{-1}(t)\right\}\right)
$$

which is an increasing function since $g\left(\left\{\mu_{k}\right\}\right)$ and each $\mu_{k}(t)$ are all increasing. The problem now is to check whether $t_{0} \in$ $\left(t^{\mathrm{lb}}\left(\left\{\tilde{L}_{k}\right\}\right), t^{\mathrm{ub}}\left(\left\{\tilde{L}_{k}\right\}\right)\right]$ exists such that $g\left(t_{0}\right)=0$. Since $g(t)$ is increasing, it suffices to check whether $g\left(t^{\mathrm{lb}}\left(\left\{\tilde{L}_{k}\right\}\right)\right)<0$ and $g\left(t^{\mathrm{ub}}\left(\left\{\tilde{L}_{k}\right\}\right)\right) \geq 0$.

We are now ready to derive a practical algorithm, which starts with the largest possible value of $t$ (largest values of the $\tilde{L}_{k}$ 's) and then decreases it until all constraints are satisfied. In more detail, Algorithm 1 first reorders the subchannels as previously described to reduce the number of hypothesis to be checked and then sets $\tilde{L}_{k}=L_{k}$ for $1 \leq k \leq N$ to obtain the initial hypothesis. At this point, if all constraints can be satisfied, i.e., if $g\left(t^{\mathrm{lb}}\left(\left\{\tilde{L}_{k}\right\}\right)\right)<0$ and $g\left(t^{\mathrm{ub}}\left(\left\{\tilde{L}_{k}\right\}\right)\right) \geq 0$, the hypothesis is accepted. Otherwise, it is rejected, and a new hypothesis has to be considered.

If a hypothesis is accepted, the optimal $t_{0}$, waterlevels $\left\{\mu_{k}\right\}$, and solution $\left\{x_{k, i}\right\}$ are readily obtained as

$$
\begin{aligned}
t_{0} & \in\left(t^{\mathrm{lb}}\left(\left\{\tilde{L}_{k}\right\}\right), t^{\mathrm{ub}}\left(\left\{\tilde{L}_{k}\right\}\right)\right] \text { such that } g\left(t_{0}\right)=0 \\
\mu_{k} & =f_{k}^{-1}\left(t_{0}\right), \quad 1 \leq k \leq N \\
x_{k, i} & =\left(\mu_{k} a_{k, i}-b_{k, i}\right)^{+}, \quad 1 \leq k \leq N, \quad 1 \leq i \leq L_{k} .
\end{aligned}
$$

If a hypothesis is rejected, a new one has to be made. We now show by induction how to do it in an efficient way such that the total number of hypotheses is reduced from $\prod_{\tilde{L}=1}^{N}\left(L_{k}+1\right)$ to $\sum_{k=1}^{N}\left(L_{k}+1\right)$. Assume that hypothesis $\left\{\tilde{L}_{k}\right\}$ has been rejected and that the optimal hypothesis (the one that contains the optimal $t$ ) is known not to contain larger values for any of the $\tilde{L}_{k}$ 's (this clearly holds for the initial hypothesis since the values of the $\tilde{L}_{k}$ 's are chosen as the highest possible). As a consequence, a new hypothesis can be made only by decreasing some of the $\tilde{L}_{k}$ 's. Noting that if any of the $\tilde{L}_{k}$ 's is decreased to some value $\tilde{L}_{k}^{\text {new }}$, then $t_{k}^{\mathrm{ub}}\left(\tilde{L}_{k}^{\text {new }}\right) \leq t_{k}^{\mathrm{lb}}\left(\tilde{L}_{k}\right)$, and also that $t_{k}^{\mathrm{lb}}\left(\tilde{L}_{k}\right) \leq t_{k_{\max }}^{\mathrm{lb}}\left(\tilde{L}_{k_{\max }}\right)$ (by definition of $k_{\max }$ ), it follows that $t_{k}^{\mathrm{ub}}\left(\tilde{L}_{k}^{\text {new }}\right) \leq t_{k_{\max }}^{\mathrm{bb}}\left(\tilde{L}_{k_{\max }}\right)$. This means that if a new hypothesis is made by decreasing some of the $\tilde{L}_{k}$ 's for $k \neq k_{\max }$, then the range of possible values for $t$ will be empty since $\left(t_{k}^{\mathrm{lb}}\left(\tilde{L}_{k}^{\text {new }}\right), t_{k}^{\mathrm{ub}}\left(\tilde{L}_{k}^{\text {new }}\right)\right] \cap\left(t_{k}^{\mathrm{lb}}\left(\tilde{L}_{k_{\max }}\right), t_{k}^{\mathrm{ub}}\left(\tilde{L}_{k_{\max }}\right)\right]=\varnothing$. Thus, we can guarantee that the optimal hypothesis must have a lower value for $\tilde{L}_{k_{\max }}$ of at most $\tilde{L}_{k_{\max }}-1$, which is taken as the next hypothesis to evaluate. Since it was assumed that the optimal hypothesis was known not to contain higher values for any of the $\tilde{L}_{k}$ 's of the original hypothesis, the new hypothesis also satisfies this condition (since it only differs in $\tilde{L}_{k_{\max }}$, and we have just shown that the optimal hypothesis has a value of $\tilde{L}_{k_{\max }}$ of at most $\tilde{L}_{k_{\max }}-1$ ). Therefore, by induction (recall that the initial hypothesis also satisfies this condition), the previous mechanism to generate new hypotheses can be repeatedly applied.

By the nature of the algorithm, the maximum number of iterations (worst-case complexity) is $\sum_{k=1}^{N}\left(L_{k}+1\right)=L_{T}+N \simeq$ $L_{T}$.

As a final remark, when evaluating a given hypothesis, note that the condition $g\left(t^{\mathrm{ub}}\left(\left\{\tilde{L}_{k}\right\}\right)\right) \geq 0$ is always satisfied in the initial hypothesis, and it is sufficient to check only the condition $g\left(t^{\mathrm{lb}}\left(\left\{\tilde{L}_{k}\right\}\right)\right)<0$. In the subsequent hypotheses, it is again sufficient to check only this condition since the other condition $g\left(t^{\mathrm{ub}}\left(\left\{\tilde{L}_{k}\right\}\right)\right) \geq 0$ is always satisfied as well (because it was implicitly evaluated in some previous rejected hypothesis).

\section{ACKNOWLEDGMENT}

The authors would like to thank S. Barbarossa for a discussion on the waterfilling interpretation that led to Fig. 2 and the anonymous reviewers for their comments on the paper.

\section{REFERENCES}

[1] R. G. Gallager, Information Theory and Reliable Communication. New York: Wiley, 1968.

[2] T. M. Cover and J. A. Thomas, Elements of Information Theory. New York: Wiley, 1991.

[3] I. E. Telatar, "Capacity of Multi-Antenna Gaussian Channels," AT\&T Bell Labs., Murray Hill, NJ, Intern. Tech. Memo, Jun. 1995.

[4] G. G. Raleigh and J. M. Cioffi, "Spatio-temporal coding for wireless communication," IEEE Trans. Commun., vol. 46, no. 3, pp. 357-366, Mar. 1998.

[5] A. Scaglione, S. Barbarossa, and G. B. Giannakis, "Filterbank transceivers optimizing information rate in block transmissions over dispersive channels," IEEE Trans. Inf. Theory, vol. 45, no. 3, pp. 1019-1032, Apr. 1999.

[6] A. Scaglione, G. B. Giannakis, and S. Barbarossa, "Redundant filterbank precoders and equalizers Part I: Unification and optimal designs," IEEE Trans. Signal Process., vol. 47, no. 7, pp. 1988-2006, Jul. 1999.

[7] D. P. Palomar, J. M. Cioffi, and M. A. Lagunas, "Joint Tx-Rx beamforming design for multicarrier MIMO channels: A unified framework for convex optimization," IEEE Trans. Signal Process., vol. 51, no. 9, pp. 2381-2401, Sep. 2003.

[8] K. H. Lee and D. P. Petersen, "Optimal linear coding for vector channels," IEEE Trans. Commun., vol. COM-24, no. 12, pp. 1283-1290, Dec. 1976.

[9] J. Salz, "Digital transmission over cross-coupled linear channels," AT\&T Tech. J., vol. 64, no. 6, pp. 1147-1159, Jul.-Aug. 1985. 
[10] J. Yang and S. Roy, "On joint transmitter and receiver optimization for multiple-input-multiple-output (MIMO) transmission systems," IEEE Trans. Commun., vol. 42, no. 12, pp. 3221-3231, Dec. 1994.

[11] H. Sampath, P. Stoica, and A. Paulraj, "Generalized linear precoder and decoder design for MIMO channels using the weighted MMSE criterion," IEEE Trans. Commun., vol. 49, no. 12, pp. 2198-2206, Dec. 2001.

[12] A. Scaglione, P. Stoica, S. Barbarossa, G. B. Giannakis, and H. Sampath, "Optimal designs for space-time linear precoders and decoders," IEEE Trans. Signal Process., vol. 50, no. 5, pp. 1051-1064, May 2002.

[13] T. Berger and D. W. Tufts, "Optimum pulse amplitude modulation. Part I: Transmitter-receiver design and bounds from information theory," IEEE Trans. Inf. Theory, vol. IT-13, no. 2, pp. 196-208, Apr. 1967.

[14] J. Yang and S. Roy, "Joint transmitter-receiver optimization for multiinput multi-output systems with decision feedback," IEEE Trans. Inf. Theory, vol. 40, no. 5, pp. 1334-1347, Sept. 1994.

[15] J. M. Cioffi and G. D. Forney, "Generalized decision-feedback equalization for packet transmission with ISI and Gaussian noise," in Coтmunications, Computation, Control and Signal Processing, A. Paulraj, V. Roychowdhury, and C. D. Schaper, Eds. Boston, MA: Kluwer, 1997, ch. 4

[16] E. N. Onggosanusi, A. M. Sayeed, and B. D. V. Veen, "Efficient signaling schemes for wideband space-time wireless channels using channel state information," IEEE Trans Veh. Technol., vol. 52, no. 1, pp. 1-13, Jan. 2003.

[17] F. Rey, M. Lamarca, and G. Vázquez, "Transmit filter optimization based on partial CSI knowledge for wireless applications," in Proc. IEEE Int. Conf. Commun., vol. 4, Anchorage, AK, May 11-15, 2003, pp. 2567-2571.

[18] Y. Ding, T. N. Davidson, Z.-Q. Luo, and K. M. Wong, "Minimum BER block precoders for zero-forcing equalization," IEEE Trans. Signal Process., vol. 51, no. 9, pp. 2410-2423, Sep. 2003.

[19] N. Amitay and J. Salz, "Linear equalization theory in digital data transmission over dually polarized fading radio channels," AT\&T Bell Labs. Tech. J., vol. 63, no. 10, pp. 2215-2259, Dec. 1984.

[20] J. M. Cioffi. (1998) EE379C Advanced Digital Communications [Online]. Available: http://www.stanford.edu/class/ee379c

[21] T. Starr, J. M. Cioffi, and P. J. Silverman, Understanding Digital Subscriber Line Technology. Upper Saddle River, NJ: Prentice-Hall, 1999.

[22] D. P. Palomar and M. A. Lagunas, "Simplified joint transmit-receive space-time equalization on spatially correlated MIMO channels: A beamforming approach," IEEE J. Sel. Areas Commun.: Special Issue on MIMO Systems and Applications, vol. 21, no. 5, pp. 730-743, Jun. 2003

[23] D. P. Palomar, M. A. Lagunas, and J. M. Cioffi, "Optimum linear joint transmit-receive processing for MIMO channels with QoS constraints," IEEE Trans. Signal Process., vol. 52, no. 5, pp. 1179-1197, May 2004.

[24] D. P. Palomar, "A Unified Framework for Communications Through MIMO Channels," Ph.D. Dissertation, Techn. Univ. Catalonia (UPC), Barcelona, Spain, May 2003.

[25] W. H. Press, S. A. Teukolsky, W. T. Vetterling, and B. P. Flannery, Numerical Recipes in C, Second ed. Cambridge, U.K.: Cambridge Univ. Press, 1992

[26] G. H. Golub and C. F. V. Loan, Matrix Computations, Third ed. Baltimore, MD: Johns Hopkins Univ. Press, 1996.

[27] A. Scaglione, S. Barbarossa, and G. B. Giannakis, "Optimal adaptive precoding for frequency-selective Nagakami-m fading channels," in Proc. IEEE 52nd Veh. Technol. Conf., vol. 3, Boston, MA, Sep. 24-28, 2000, pp. 1291-1295.

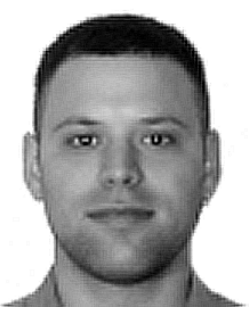

Daniel Pérez Palomar (S'99-M'03) received the Electrical Engineering and Ph.D. degrees from the Technical University of Catalonia (UPC), Barcelona, Spain, in 1998 and 2003, respectively.

During 1998, he was with the Department of Electronic Engineering, King's College London (KCL), London, U.K. From January 1999 to December 2003, he was a Research Assistant with the Department of Signal Theory and Communications, UPC. From April to November 2001, he held a visiting research appointment at the Department of Electrical Engineering, Stanford University, Stanford, CA. From January to December 2002, he was a visiting researcher with the Telecommunications Technological Center of Catalonia (CTTC), Barcelona. From August to November 2003, he was a Guest Researcher with the Department of Signals, Sensors, and Systems, Royal Institute of Technology (KTH), Stockholm, Sweden. From November 2003 to February 2004, he was a Visiting Researcher with the INFOCOM Department, University of Rome "La Sapienza," Rome, Italy. He is currently a Fulbright Research Fellow at Princeton University, Princeton, NJ. He has participated in several European projects such as ACTS-SUNBEAM (1999), IST-METRA (2000-2001), IST I-METRA (2001-2003), and IST ROMANTIK (2002-2004). His primary research interests include information-theoretic and communication aspects of wireless MIMO channels and array signal processing, with special emphasis on convex optimization theory applied to communications systems.

Dr. Palomar received the 2002-2003 Rosina Ribalta first prize for the Best Doctoral Thesis within the areas of information technologies and communications from the Epson Foundation. He also received the 2003 Prize fo rthe Best Doctoral Thesis in advanced mobile communications from the Vodaphone Foundation and COIT. He has also received a Fulbright Research Fellowship.

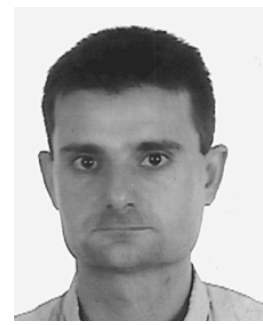

Javier Rodríguez Fonollosa (S'90-M'92-SM'98) received the telecommunication engineering degree from the Technical University of Catalunya (UPC), Barcelona, Spain, in 1988 and the Ph.D. degree in electrical and computer engineering from Northeastern University, Boston, MA, in 1992

In 1993, he joined the Department of Signal Theory and Communications, UPC, where he became Associate Professor in 1996 and Professor in 2003 . He is the author of 15 journal and more than 70 conference papers in the area of signal processing and communications. Since 1995, he lead UPC's participation in the European Commission-funded ACTS Mobile projects TSUNAMI(II) and SUNBEAM, which included the analysis of adaptive antennas in second- and third-generation cellular communication systems. In January 2000, he acted as technical and project coordinator of the IST project METRA, which was dedicated to the introduction of multiantenna terminals in UMTS, and which continued until 2003 under the name of I-METRA, looking into Systems beyond third generation. His research interests include many different aspects of statistical signal processing for communications.

Dr. Fonollosa was co-chairman and organizer of the IEEE Signal Processing/ATHOS Workshop on Higher Order Statistics, Begur, Girona, Spain, in June 1995. He was elected member of the Signal Processing for Communications (SPCOM) Technical Committee of the IEEE Signal Processing Society in January 1999. He participated as a panelist in the National Science Foundation evaluation committee on Wireless Information Technology and Networking in 1999 and for the European Commission for the evaluation of proposals and technical verification of projects submitted to the Fifth Framework Programme in 2001 and 2002. Since April 2003, he has been an evaluator for the European Commission in the Future and Emerging Technologies area of the Sixth Framework Programme. 